ELECTRONIC RESEARCH ANNOUNCEMENTS OF THE AMERICAN MATHEMATICAL SOCIETY

Volume 5, Pages 66-70 (May 28, 1999)

S $1079-6762(99) 00062-1$

\title{
THE HILBERT-SMITH CONJECTURE FOR QUASICONFORMAL ACTIONS
}

\author{
GAVEN J. MARTIN
}

(Communicated by Walter Neumann)

\begin{abstract}
This note announces a proof of the Hilbert-Smith conjecture in the quasiconformal case: A locally compact group $G$ of quasiconformal homeomorphisms acting effectively on a Riemannian manifold is a Lie group. The result established is true in somewhat more generality.
\end{abstract}

\section{INTRODUCTION}

This note announces a proof of the Hilbert-Smith conjecture for quasiconformal actions on Riemannian manifolds and related spaces.

We were led to this conjecture through our study of two important areas of geometric function theory. The first concerns the problem of unique analytic continuation of solutions to general Beltrami systems in higher dimensions:

$$
D f^{t}(x) G(f(x)) D f(x)=J_{f}(x)^{2 / n} H(x) \quad \text { a.e., }
$$

where $G$ and $H$ are bounded measurable functions valued in the space of symmetric positive definite $n \times n$ matrices of determinant 1 ( $D f$ is the Jacobian matrix of $f$, and $J_{f}$ its determinant). Solutions to these nonlinear systems of PDE's are quasiregular or quasiconformal mappings. These mappings play an important role in many aspects of modern geometry and analysis. For instance, in nonlinear elasticity, topological index theory, harmonic analysis, nonlinear potential theory, and so forth. These mappings are natural higher dimensional analogues of analytic or conformal mappings in $\mathbb{C}$. See [11] for a good starting point to the theory. The second problem we were concerned with was the classification of the dynamics of mappings which are rational with respect to a bounded measurable Riemannian stucture, now called uniformly quasiregular mappings. This theory was initiated in [4], but see [6] too. Largely because of Rickman's version of Montel's normal families criterion, this theory closely parallels the classical iteration theory of rational endomorphisms of $\overline{\mathbb{C}}$, but again in higher dimensions. We were interested in proving Siegel's theorem concerning the local conjugacy of rotational dynamics about nonattracting fixed points in the Fatou set.

How these problems are related and how their affirmative solution follows from the Hilbert-Smith conjecture is discussed in [5], where more details can be found for the sketch of proof of our main results given here.

Received by the editors November 9, 1998.

1991 Mathematics Subject Classification. Primary 26A24, 30C60, 53A04, 54F65.

Research supported in part by a grant from the N.Z. Marsden Fund.

(C)1999 American Mathematical Society 
Hilbert's fifth problem [3] asks if every finite dimensional locally Euclidean topological group is necessarily a Lie group. This problem was solved by von Neumann in 1933 for compact groups and by Gleason and Montgomery and Zippin in 1952 for locally compact groups; see [8] and the references therein.

A more general version of the fifth problem asserts that among all locally compact groups $G$ only Lie groups can act effectively on finite dimensional manifolds. This problem has come to be called the Hilbert-Smith Conjecture. It follows from the work of Newman and of Smith together with the structure theory of infinite abelian groups that the conjecture is equivalent to the special case when the group $G$ is isomorphic to the $p$-adic integers $A_{p}$.

In 1943 Bochner and Montgomery [1] solved this problem for actions by diffeomorphisms. Although there is considerable literature on this problem, for our purposes the fundamental result we need was established by Yang [13] in 1960.

Theorem 1.1. If $G$ is a p-adic group acting effectively on a homology n-manifold $M$, then the orbit space $M / G$ is of homological dimension $n+2$.

Yang's result is based on the construction of certain exact sequences in a modified version of Smith homology theory and might nowadays be regarded as standard. There are relatively nice $n$-dimensional metric spaces on which the $p$-adics can act increasing the dimension [9].

It took until 1997 for Yang's result to be used in an effective way. Repovš and Ščpin used it to prove the Hilbert-Smith Conjecture for Lipschitz actions by comparing the Hausdorff and cohomological dimensions of the orbit space, [10]. R. D. Edwards has announced work toward a solution of the Hilbert-Smith conjecture for free actions.

Once we became aware of the work [10], it became clear how one might establish the result in the more general setting of quasiconformal mappings. There are technical difficulties because quasiconformal mappings are not Lipschitz and although they enjoy Hölder continuity properties, these are not sufficient to directly apply the method of Repovš and Ščepin. We must thank K. Astala for pointing out how to make an important step in our argument. Our main results are as follows. We give relevant definitions and a sketch of proof in the next sections.

Theorem 1.2. Let $G$ be a locally compact group acting effectively and quasiconformally on a Riemannian manifold. Then $G$ is a Lie group.

As noted above, this result is implied by the following

Theorem 1.3. The group $A_{p}$ of $p$-adic integers cannot act effectively by quasiconformal homeomorphisms on any Riemannian manifold.

Remark. Sullivan has shown that every topological $n$-manifold $(n \neq 4)$ admits a unique quasiconformal structure, [12]. (The situation in 4 dimensions is more complicated; see [2].) Thus it is possible to speak of quasiconformal actions on an arbitrary topological $n$-manifold, $n \neq 4$. It is in this setting that our results hold and thus the hypothesis that the manifold is Riemannian is largely unnecessary. However, if $G$ is a compact group acting effectively on a topological manifold, it is far from clear that the action can be made quasiconformal (indeed this would solve the general Hilbert-Smith conjecture $(n \neq 4)$ in view of our result). 


\section{QuASICONFORMAL MAPPINGS}

Let $\Omega$ and $\Omega^{\prime}$ be open subsets of $\overline{\mathbb{R}}^{n}$, and let $f: \Omega \rightarrow \Omega^{\prime}$ be a homeomorphism. Quasiconformal mappings are first and foremost mappings of bounded distortion. The infinitesimal distortion of $f$ at $x$ is

$$
H_{f}(x)=\limsup _{r \rightarrow 0} \frac{\max _{|h|=r}|f(x+h)-f(x)|}{\min _{|h|=r}|f(x+h)-f(x)|} .
$$

Definition. A homeomorphism $f: \Omega \rightarrow \Omega^{\prime}$ between subdomains of $\mathbb{R}^{n}$ is quasiconformal if there is $H<\infty$ such that

$$
H_{f}(x) \leq H \quad \text { for all } x \in \Omega
$$

The essential supremum of the function $H_{f}(x)$ is called the maximal distortion or dilatation of $f$. Unfortunately, this definition, while aesthetically pleasing, is difficult to work with. Also it is not lower semicontinuous with repect to limits, a property we shall need. It is more usual to study quasiconformal mappings as Sobolev functions satisfying the differential inequality between the Jacobian matrix $D f$ and its determinant $J_{f}$,

$$
|D f(x)|^{n} \leq K J_{f}(x) \quad \text { a.e. in } \Omega .
$$

We say that a $W_{l o c}^{1, n}\left(\Omega, \mathbb{R}^{n}\right)$ function satisfying this inequality $f$ is $K$-quasiconformal. There are other natural definitions involving distortion of moduli (or other conformal invariants), and an important part of the foundations of the theory is the equivalence of these definitions. The generalisations to quasiconformal mappings between quasiconformal manifolds and so forth are straightforward (see [11]). It is also clear that Lipschitz and smooth maps are locally quasiconformal and therefore quasiconformal on relatively compact subdomains.

Definition. A topological transformation group $G$ acting on a quasiconformal $n$ manifold $M$ is said to act quasiconformally if each $g \in G$ is quasiconformal.

Remark. It is important to note that we have made no a priori assumption of a uniform bound on the dilatation of each element of $G$.

\section{Sketch of PRoof For Theorem 1.3}

We break the proof into a number of steps.

1. It is enough to consider the $p$-adics acting effectively and quasiconformally as a transformation group $G$ of a domain $\Omega$ in $\mathbb{R}^{n}$ (the ideas are the same). For a positive integer $n$ we set

$$
E_{n}=\left\{g \in G: K_{g} \leq n\right\}
$$

Then $E_{n}$ is a countable family of closed sets whose union is $G$. By the Baire category theorem there is some $E_{n}$ with nonempty interior. Translating by an element of $G$ we observe that there is some $m \geq 0$ with $E_{m}$ containing a neighbourhood of the identity. Any neighbourhood of the identity in the $p$-adic group contains an isomorphic copy of the $p$-adics and so, after replacing $G$ with this copy, we may assume that there is $K<\infty$ such that each $g \in G$ is $K$-quasiconformal. That is, $G$ is a uniformly quasiconformal group. 
2. $G$ has an invariant Haar measure $d g$ of total mass 1. Point evaluation $x \mapsto g(x)$ is continuous on $G$ and thus we may construct an invariant metric on $\Omega$ as

$$
d_{G}(x, y)=\int_{G}|g(x)-g(y)| d g .
$$

This metric generates the usual topology of $\Omega$. In fact, since each $g \in G$ is now locally $1 / K$-Hölder continuous, the invariant metric is locally Hölder equivalent to our background metric. We want to estimate the Hausdorff dimension of the metric space $\left(\Omega, d_{G}\right)$. This is a local problem. The compactness of $G$ allows us to assume $\operatorname{Vol}(\Omega)<\infty$. Let $r$ be a small number and cover a relatively compact open subset $U$ of $\Omega$ by a family $\mathcal{F}$ of balls of radius $r$ in the background metric. We may refine this cover using the Besicovitch covering theorem [7] so as to have bounded overlap (independent of $r$ ). That is, there is a fixed constant $c$ such that each $B \in \mathcal{F}$ meets at most $c$ other elements of $\mathcal{F}$. We want to look at the sum

$$
\sum_{B \in \mathcal{F}} d_{G}(B)^{n}
$$

where $d_{G}(B)$ is the diameter of $B$ in the invariant metric, and bound it by a number independent of $r$. This will imply that the Hausdorff dimension of the space $\left(\Omega, d_{G}\right)$ is at most $n$. For each $B \in \mathcal{F}$ let $x_{B}$ denote its center and $y_{B}$ a point on the boundary with $d_{G}(B) \leq 2 d_{G}\left(x_{B}, y_{B}\right)$. Then

$$
\begin{aligned}
& \sum_{B \in \mathcal{F}} d_{G}(B)^{n} \\
& \leq C_{n} \sum_{B \in \mathcal{F}}\left(\int_{G}\left|g\left(x_{B}\right)-g\left(y_{B}\right)\right| d g\right)^{n} \\
& \leq C_{n} \sum_{B \in \mathcal{F}} \int_{G}\left|g\left(x_{B}\right)-g\left(y_{B}\right)\right|^{n} d g \quad \text { (Jensen's inequality) } \\
& \leq C_{n, K} \sum_{B \in \mathcal{F}} \int_{G} \min _{|h|=r}\left|g\left(x_{B}\right)-g\left(x_{B}+h\right)\right|^{n} d g \quad(K \text {-quasiconformality }) \\
& \leq C_{n, K} \sum_{B \in \mathcal{F}} \int_{G} \operatorname{Vol}(g(B)) d g=C_{n, K} \int_{G} \sum_{B \in \mathcal{F}} \operatorname{Vol}(g(B)) d g \\
& \leq C_{n, K} \operatorname{Vol}(\Omega) \quad \text { Balls have bounded overlap). }
\end{aligned}
$$

We have used the usual convention that the constant may change from line to line, but have indicated its dependence on the parameters. The quasiconformal fact used is essentially Mori's theorem and the reduction to the uniformly quasiconformal case is crucial here. We have now shown that $\left(\Omega, d_{G}\right)$ has Hausdorff dimension $n$.

3. As the metric $d_{G}$ is invariant, the orbit space $\Omega / G$ inherits the metric $\rho(\eta, \zeta)=$ $d_{G}(G(x), G(y))$, where $\eta=\pi(x)$ and $\pi: \Omega \rightarrow \Omega / G$ is the projection. The map $\pi$ is evidently a contraction. Hausdorff dimension cannot increase under a contraction mapping. Therefore the Hausdorff dimension of $\Omega / G$ is at most $n$. We now use a few simple facts from dimension theory. First, the Hausdorff dimension dominates the topological dimension, which in turn then dominates the cohomological dimension. The result now follows as we have obtained a contradiction to Yang's theorem. 


\section{REFERENCES}

[1] S. Bochner and D. Montgomery, Locally compact groups of differentiable transformations, Ann. Math. 47 (1946), 639-653. MR 8:253c

[2] S. Donaldson and D. Sullivan, Quasiconformal 4-manifolds, Acta Math. 163 (1989), 181252. MR 91d:57012

[3] D. Hilbert, Mathematische Probleme, Nachr. Akad. Wiss. Göttingen (1900), 253-297.

[4] T. Iwaniec and G. J. Martin, Quasiregular semigroups, Ann. Acad. Sci. Fenn. Math. 21 (1996), 241-254. MR 97i:30032

[5] G. J. Martin, UQR mappings, Siegel's theorem and the Hilbert-Smith conjecture, in preparation.

[6] V. Mayer, Uniformly quasiregular mappings of Lattès type, Conformal Geometry and Dynamics 1 (1997), 24-27. MR 98j:30017

[7] G.V. Maz'ja, Sobolev spaces, Springer-Verlag, 1985. MR 87g:46056

[8] D. Montgomery and L. Zippin, Topological transformation groups, Interscience, New York, 1955. MR 17:383b

[9] F. Raymond and R. F. Williams, Examples of p-adic transformation groups, Ann. Math. 78 (1963), 92-106. MR 27:756

[10] D. Repovš and E.V. Ščepin, A proof of the Hilbert-Smith conjecture for actions by Lipschitz maps, Math. Annalen 2 (1997), 361-364. MR 99c:57066

[11] S. Rickman, Quasiregular mappings, Springer-Verlag, 1993. MR 95g:30026

[12] D. Sullivan, Quasiconformal and Lipschitz structures, to appear.

[13] C-T. Yang, p-adic transformation groups, Michigan Math. J. 7 (1960), 201-218. MR 22:11065

Department of Mathematics, The University of Auckland, Private Bag 92019, AuckLAND, New Zealand

E-mail address: martin@math.auckland.ac.nz 